OPEN ACCESS

Edited by:

Nianyong Chen,

Sichuan University, China

Reviewed by:

Jennifer Rosenberg,

Penn State Milton S. Hershey Medical

Center, United States

Valentina Lancellotta,

Catholic University of the Sacred

Heart, Italy

*Correspondence:

Guang-Hui Cheng

chenggh@jlu.edu.cn

Specialty section:

This article was submitted to

Radiation Oncology,

a section of the journal

Frontiers in Oncology

Received: 26 October 2020 Accepted: 30 December 2020 Published: 18 February 2021

Citation:

Qu H-D, Han D-M, Zhang N, Mao Z and Cheng G-H (2021) Intracavitary/ Interstitial Applicator Plus Distal

Parametrial Free Needle Interstitial Brachytherapy in Locally Advanced Cervical Cancer: A Dosimetric Study.

Front. Oncol. 10:621347.

doi: 10.3389/fonc.2020.621347

\section{Intracavitary/Interstitial Applicator Plus Distal Parametrial Free Needle Interstitial Brachytherapy in Locally Advanced Cervical Cancer: A Dosimetric Study}

\author{
Hong-Da Qu, Dong-Mei Han, Ning Zhang, Zhuang Mao and Guang-Hui Cheng * \\ Department of Radiation Oncology, China-Japan Union Hospital of Jilin University, Changchun, China
}

Purpose: To explore the dosimetric advantage of combining intracavitary/interstitial applicator with distal parametrial free needle interstitial brachytherapy (IC/IS+ISBT DP) based on MRI for locally advanced cervical cancer.

Methods and Materials: 77 IC/IS+ISBT DP treatment plans were developed for 34 patients with locally advanced cervical cancer from June 2016 to January 2020 in this study. We removed the free needles and devised a new IC/ISBT treatment plan based on the same principle. We then compared the dosimetric differences of D90, D98, V100, V150, V200 for HR-CTV (high-risk clinical target volume), D90 for IR-CTV (Intermediate risk-CTV) and D2cc for OARs (organs at risk) between the two groups of treatment plans for the same patient, and the paired T test was performed in parallel. Further, the dosage differences between the two group plans under different parametrial extension widths (the maximum distance of HR-CTV from the vertical direction of the uterine tandem at coronal position) were compared. The survival rate was calculated using the Kaplan-Meier method. Prognostic factors for overall survival (OS) and progression-free survival (PFS) were determined by Cox regression method. RTOG/EORTC criteria were used to grade toxicities.

Results: A total of 297 free needles were used, with a weight ratio of $15.8 \% \pm 0.11$, and a mean insertion depth of $6.52 \mathrm{~cm} \pm 2.8 \mathrm{~cm}$. D90, D98, V100 for HR-CTV, and D90 for IRCTV for IC/IS+ISBT DP were significantly higher than IC/ISBT for which free needles were removed $(p<0.05)$. And the V200 for HR-CTV and D2cc for bladder, rectum and sigmoid were decreased $(p<0.05)$. When the parametrial extension widths were greater than $3 \mathrm{~cm}$, the HR-CTV D90 and the D2CC for rectum, bladder and sigmoid colon for IC/IS-ISBT DP were advantageous compared to IC/ISBT ( $p<0.05)$. The 2-yr OS, PFS and local control rate $(\mathrm{LC})$ were $82.3,66.8$, and $93.1 \%$, respectively. Parametrial extension widths was the only statistically prognostic factors for PFS $(p=0.002)$ on univariate analysis. No grade 3 or 4 Treatment-related toxicities were observed. 
Conclusion: Our institutional experiences showed that IC/IS+ISBT DP is an effective treatment for cervical cancer patients with distal parametrial extension. IC/IS-ISBT DP had dosage advantage and clinical feasibility in locally advanced cervical cancer with distal parametrial extension when the parametrial extension widths were greater than $3 \mathrm{~cm}$.

Keywords: cervical cancer, interstitial brachytherapy, dosimetric analysis, treatment outcome, parametrial interstitial brachytherapy

\section{INTRODUCTION}

The combination of external beam radiotherapy (EBRT), concurrent chemotherapy and brachytherapy (BT) is the standard treatment for locally advanced cervical cancer, among which brachytherapy is indispensable $(1,2)$. Brachytherapy can be divided into intracavity (ICBT), interstitial (ISBT) and combined intracavitary/interstitial brachytherapy (IC/ISBT). ICBT has been used for decades and is most suitable for smallvolume and superficial lesions. However, when the tumor is bulky, eccentrically located or irregular in shape, problems can arise such as poor target volume coverage, insufficient dose, or excessive radiation to organs at risk (OAR). To avoid these potential problems, IC/ISBT was developed, which can increase the fitness of target volume and the uniformity of dose distribution (3). Nevertheless, the dose coverage of IC/ISBT in cervical cancer with distal parametrial extension remained limited (4). For improved parametrial coverage, free needles have been implanted into distal parametrial tumor extensions in combination with IC/ISBT. However, the dosimetry of this combined technique has rarely been reported. The purpose of this study was to investigate the dosimetric advantages of IC/IS+ ISBT DP for locally advanced cervical cancer with distal parametrial extension, and to report the result of this radiation technique.

\section{MATERIALS AND METHODS}

\section{Patient Selection and Treatment}

The study comprised 34 patients with locally advanced cervical carcinoma, for whom 77 IC/IS+ISBT DP treatment plans were developed, starting in June 2016 and extending to January 2020. The average age was 53 years (range, $36-76$ years), with a median age of 54 years. The pathology diagnoses included 32 cases of squamous cell carcinoma, and 2 cases of adenocarcinoma. Disease staging for patients admitted prior to September 2018 was based on the 2009 FIGO system, and patients admitted after September 2018 were based on the 2018 FIGO system. All patients were free of distant metastasis prior to treatment. And 7 patients had received 1-3 cycles of neoadjuvant chemotherapy (TP), but still inoperable, were transferred to our department to receive definitive radio(chemo)therapy. All patients were treated with EBRT (45 Gy in 25 fractions), administered via intensity modulated radiation therapy (IMRT) or three dimensional conformal radiotherapy (3D-CRT). Patients with pathology or imaging-positive lymph nodes received an increased dose of 60
Gy. Platinum-based single-drug or dual-drug concurrent chemotherapy was administered during the EBRT. Single-agent cisplatin was administered at a dose of $30-40 \mathrm{mg} / \mathrm{m}^{2}$ once a week for 5 weeks. Dual-drug concurrent chemotherapy $\left(60 \mathrm{mg} / \mathrm{m}^{2}\right.$ cisplatin $+60 \mathrm{mg} / \mathrm{m}^{2}$ docetaxel or $135 \mathrm{mg} / \mathrm{m}^{2}$ paclitaxel every three weeks for 3 cycles) was considered for patients with largevolume tumor in order to control micrometastasis as much as possible, although those patients who could not tolerate chemotherapy were treated with radiotherapy alone.

Gynecologic physical examination and pelvic MRI were performed within one week prior to initiating brachytherapy. The appropriate applicator was selected according to the patient's tumor regression. The 77 treatment plans used IC/IS+ ISBT DP to include as much of the distal parametrial extension as possible.

\section{Applicator Implantation Method and Dose Calculation}

MRI-guided high-dose-rate (HDR) 192Ir BT (Micro-Selectron HDR) was performed for all patients.

The implantation process proceeded according to the following description. Prior to the operation, different angles of the uterine tandem were chosen according to the position of the uterus. The tumor location, size, shape, parametrial invasion and the relationship with the surrounding organs were derived from the MRI images before and after EBRT, as well as the gynecological examination. These guided the selection of the appropriate needle position, implantation depth, the depth from the perineum to the tumor, and the route of needle insertion. Preoperatively, the patient underwent vaginal irrigation, urinary indwelling catheterization and enema preparation. Under general anesthesia, the applicator (Utrecht/circular applicator) was implanted under real-time ultrasound guidance in accordance with the preoperative preset; the depth of the needle in the insertion points could be adjusted utilizing ultrasound guidance. Evaluate the distance of HR-CTV from the lateral edge of the parametrium to the needles in the fixed insertion points combined with ultrasound on transverse position. If the distance exceeds $1.3-1.5 \mathrm{~cm}$, carefully consider whether to increase the free needles on distal parametrium (the Near Maximum Distance approximately exceed 3-3.2cm). Upon completion of the implantation, with needles fixed, the rectal pressure plate was used to push open the rectum. The prescription dose delivered was $7 \mathrm{~Gy} \times 4 \mathrm{f}, 1$ week/fraction.

Magnetic resonance images were obtained by $3.0 \mathrm{~T}$ MRI scanner after the implantation was completed. Images include sagittal, axial and coronal T2-weighted turbo spin-echo images, 
with a slice thickness of $4.5 \mathrm{~mm}$ and a balanced steady-state free precession scan with $1.5 \mathrm{~mm}$ slice thickness for applicator reconstruction purposes.

\section{Contouring and Treatment Planning}

HR-CTV and IR-CTV were contoured based on MRI and clinical examination before and after EBRT, according to the recommendation of the GEC-ESTRO (5). Contoured OARs included the bladder, rectum, sigmoid and small bowel. We contour the HR-CTV as the entire cervix and any notable remaining tumor at BT (5). Specific borders for HR-CTV were delineated according to the recommendation of Viswanathan et al. (6). In general, the lower border covered the inferior cervical margin. For disease that extended to the vagina, the lower border was determined through clinical examination. Superiorly, the HR-CTV was contoured at the level where the uterine cavity appeared, further extending $1 \mathrm{~cm}$ upwards to form a cone-shaped tip. To encompass intrauterine disease, the HRCTV was defined by sagittal T2-weighted MRI images. Any lateral parametrial disease extension required contouring based on the combination of clinical gynecologic examination and MRI images. The IR-CTV represents the initial gross tumor volume (GTVinit) as superimposed on the topography at the time of brachytherapy, together with a margin surrounding the HR-CTV in areas without an initial GTVinit. By definition, the IR-CTV includes all of the HR-CTV and safety margins as appropriate.

\section{Plan Design and Evaluation}

Under the guidance of MRI, the applicator and the implant needles were reconstructed in the treatment planning system (TPS), and the source dwell point of the radioactive source was selected according to the shape of the target area and the relative, three dimensional positional relationship of the OARs. We optimized the treatment plan based on the prescribed dose and OARs dose limits. The final objective was a total dose (combined EBRT and BT) of HR-CTV D90 $\geq 85$ Gy, IR-CTV D90 $\geq 60$ Gy, bladder D2cc $\leq 80 \mathrm{~Gy}$, and rectum/sigmoid/small bowel D2cc $\leq$ 70 Gy. Using the linear-quadratic (LQ) model to calculate the biologically equivalent dose in 2 Gy fractions (EQD2), normal tissue $\alpha / \beta=3$, tumor $\alpha / \beta=10$, the formula is as follows (7):

$$
E Q D 2=\frac{N d\left(1+\frac{d}{\alpha / \beta}\right)}{1+\frac{2}{\alpha / \beta}}
$$

Each fractional treatment plan of IC/IS+ISBT DP group generated a simulation plan leaving the free needles, defined as the IC/ISBT research group. However, this optimized IC research plan was not used clinically. The aim dose to be delivered to the target and the limit dose to the OAR in the IC/ISBT research plan were the same as the criteria of the IC/IS+ISBT DP group.

\section{Follow-Up}

All patients were clinically evaluated by imaging studies (MRI or CT scan) and bimanual pelvic examination at the first 1 month and once every three months during the first 2 years after the completion of all treatments, and every 6 months thereafter. The
OS time was calculated from the date of treatment start to the date of death or last follow up. The PFS time was calculated from the date of starting treatment to the date of disease progression, relapse, disease-related death, or the last follow-up. The LC time was calculated from the date of starting treatment to the date of local recurrence or last follow up.

CR were defined as a $100 \%$ decrease in gross tumors. And we defined partial response (PR) as $\geq 50 \%$ decrease of primary gross tumor. Treatment response was evaluated by imaging studies (MRI or CT scan) and bimanual pelvic examination at 3 months after completion of brachytherapy. RTOG/EORTC criteria are used to grade toxicities (8).

\section{Data Processing and Statistical Analysis}

The height, width, thickness, volume, and the near maximum distance from uterine tandem (NMD, Figure 1) of the treatment volume (TV, target area covered by the $100 \%$ isodose curve) between the two groups was compared using paired t-test. Likewise, the paired t-test was performed on the HR-CTV D90, D98, V100, V150, V200, IR-CTV D90, and OAR D2cc of these two groups before and after. Furthermore, we compared dosimetric differences for the different parametrial extension widths between these two groups. OS, PFS and LC were calculated using the Kaplan-Meier method. Potential prognostic factors for OS and PFS were investigated by using univariate Cox regression models. Differences were considered significant at $\mathrm{P}<0.05$ (2-sided). All statistical analyses were performed using GraphPad Prism 8 Software (GraphPad Software, Inc, San Diego, CA).

\section{RESULTS}

\section{Patient Characteristics}

All 34 patients completed EBRT with 45 Gy/25 f and received brachytherapy $28 \mathrm{~Gy} / 4 \mathrm{f}$ in our department. Characteristics for 34 patients are shown in Table 1.

\section{Characteristics of Brachytherapy}

A total of 77 IC/IS+ISBT DP fractions were administered to 34 patients using 1054 needles, including 297 free needles. The mean number of free needles was 3.86 (range, 1-9). The weight ratio of these free needles was $15.8 \% \pm 0.11$, and the mean insertion depth was $6.52 \mathrm{~cm} \pm 2.8 \mathrm{~cm}$.

\section{Volumetric Comparison}

We compared the volumetric of the IC/IS+ISBT DP treatment plan with the IC/ISBT treatment plan for which the free needles had been removed. The comparison results of the height, width, thickness, volume and NMD of TV between the two treatment plans are shown in Table 2. The width, NMD and volume of the TV IC/IS+ISBT DP group are significantly greater than the IC/ ISBT group, while the thickness was slightly lower $(p<0.05)$.

\section{DVH Parameters}

The comparison results of the dose-volume histogram (DVH) parameters of these two treatment plans are listed in Table 3. 


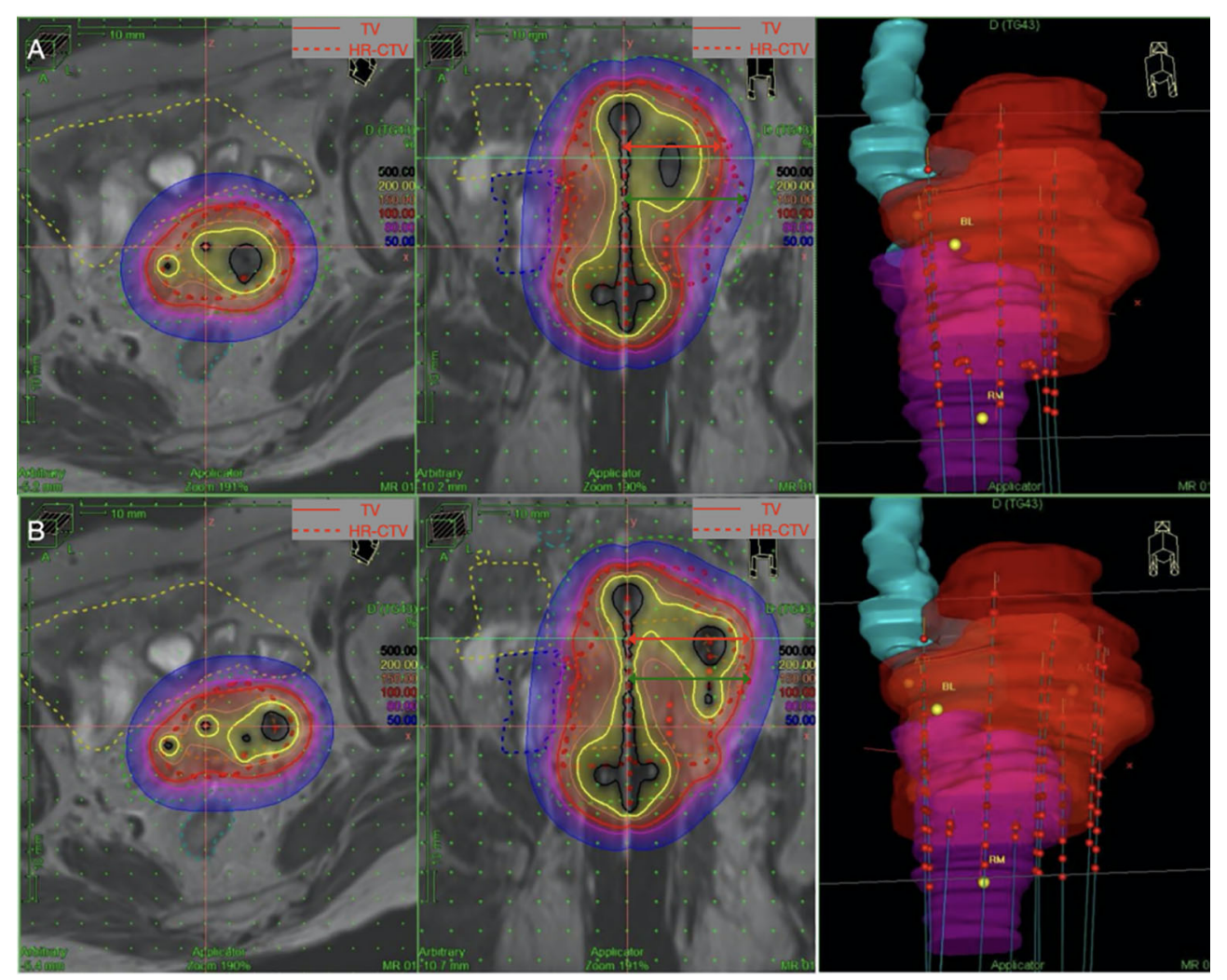

FIGURE 1 | NMD (red arrow) is defined as the maximum distance of the 100\% isodose curve from the vertical direction of the uterine tandem at coronal position. Parametrial extension widths (green arrow) is defined as the maximum distance of HR-CTV from the vertical direction of the uterine tandem at coronal position. The parametrial extension widths of this patient is $4.06 \mathrm{~cm}$. (A) The standard loading of the IC/ISBT for which free needles were removed shows insufficient coverage of the HR-CTV (D90: 6.06Gy, V100: 83.21\%,NMD:3.18cm). (B) Loading of the free needles on the left improves the coverage of the HR-CTV(D90:7.15Gy,

V100:91.66\%,NMD:4.19cm). CTV, clinical target volume; HR, high risk; D90, the minimum dose delivered to $90 \%$ of the target volume. TV, treatment volume, target area covered by the $100 \%$ isodose curve.

Notably, the D90, D98, V100 of HR-CTV and D90 of IR-CTV for the IC/IS+ISBT DP group were significantly higher than that of the IC/ISBT group, while the HR-CTV V200 was slightly lower $(p<0.05)$. Regarding the protection of OARs, all treatment plans met the single dose limit (bladder D2cc $<5.88$ Gy; rectum, sigmoid colon and small intestine D2cc $<4.98$ Gy). Importantly, the D2cc of the bladder, rectum and sigmoid of IC/IS+ISBT DP were lower than IC/ISBT $(\mathrm{p}<0.05)$.

\section{Dosimetric Comparison Under Different Parametrial Extension Widths}

All 77 treatment plans were divided into 4 groups according to the parametrial extension widths of $\leq 3,3.1-3.5,3.6-4.0$, and $>4 \mathrm{~cm}$, and compared the HR-CTV D90 of IC/IS+ISBT DP to the IC/ISBT treatment plans (Table 4). The HR-CTV D90 of IC/IS+ISBT DP was significantly higher than the IC/ISBT treatment plan for parametrial extension widths exceeding $3 \mathrm{~cm}(p<0.05)$. Furthermore, we divided all treatment plans into 2 groups based on the parametrial extension width of $\leq 3 \mathrm{~cm}$, and performed paired t-tests on the $\mathrm{D}_{2 \mathrm{CC}}$ of the relevant OAR between IC/IS+ISBT DP and IC/ISBT treatment plans (Table 5). Although the $\mathrm{D}_{2 \mathrm{CC}}$ of bladder and small bowel showed no significant difference, the $\mathrm{D}_{2 \mathrm{CC}}$ of rectum, bladder, and sigmoid for IC/IS+ISBT DP showed dosimetry advantage for parametrial extension widths exceeding $3 \mathrm{~cm}(p<0.05)$.

\section{Treatment Outcomes}

Eighteen patients (52.9\%) had CR and 16 patients $(47.1 \%)$ had PR. The median follow-up period for 34 patients was 710 days (range, 241-1696) with 9 cases $<1$ year; 11 cases $\geq 1$ year, $<2$ years; 7 cases $\geq 2$ years, $<3$ year; 7 cases $\geq 3$ years. Patients received a median of 91.15GyEQD2 for D90 HR-CTV. The 2-yr OS, PFS and local control rate (LC) were $82.3 \%, 66.8 \%$ and $93.1 \%$, respectively (Figure 2). Treatment failure were experienced in nine patients during the follow-up: of which 2 patients had local recurrence and 7 had distant metastasis. In univariate analysis (Table 6), parametrial extension widths ( $<3 \mathrm{vs} . \geq 3 \mathrm{~cm}, \mathrm{p}=0.002$ ) showed a significant effect on PFS (Figure 3).

\section{Treatment-Related Toxicities}

Complications were limited to 2 cases of local infection during implantation, and were treated successfully with a single course of antibiotics. No serious bleeding or transfusions requirements 
TABLE 1 | Patient characteristics and treatment parameters.

\begin{tabular}{|c|c|}
\hline Characteristic & Value \\
\hline Age at primary diagnosis (years) & Median:54 (range: 36-76) \\
\hline \multicolumn{2}{|l|}{ Tumor stage (FIGO2009) } \\
\hline$\| \mathrm{B}$ & 9 \\
\hline$\| I A$ & 2 \\
\hline IIIB & 10 \\
\hline IVA & 1 \\
\hline \multicolumn{2}{|l|}{ Tumor stage (FIGO2018) } \\
\hline IIB & 1 \\
\hline Illc1 & 7 \\
\hline IIIB & 3 \\
\hline IVA & 1 \\
\hline \multicolumn{2}{|l|}{ Neoadjuvant chemotherapy } \\
\hline $\mathrm{TP}$ & 7 \\
\hline none & 27 \\
\hline \multicolumn{2}{|l|}{ Concurrent chemotherapy } \\
\hline $\mathrm{TP}$ & 1 \\
\hline $\mathrm{DP}$ & 6 \\
\hline Single-drug platinum & 18 \\
\hline none & 9 \\
\hline \multicolumn{2}{|l|}{ Sequential chemotherapy } \\
\hline TP & 7 \\
\hline none & 27 \\
\hline Follow up (day) & Median: $710(241-1,696)$ \\
\hline HR-CTV D90 (Gy) & $91.15 \pm 4.06$ \\
\hline IR-CTV D90 (Gy) & $66.64 \pm 3.74$ \\
\hline Bladder D2cc (Gy) & $78.88 \pm 6.02$ \\
\hline Rectem D2cc (Gy) & $67.31 \pm 5.25$ \\
\hline Sigmoid D2cc (Gy) & $64.30 \pm 6.35$ \\
\hline small bowel D2cc (Gy) & $62.02 \pm 6.30$ \\
\hline \multicolumn{2}{|l|}{ Treatment outcome } \\
\hline $\mathrm{CR}$ & 18 \\
\hline PR & 16 \\
\hline
\end{tabular}

FIGO, International Federation of Gynecology and Obstetrics.

TP, cisplatin plus paclitaxel; DP, Cisplatin plus docetaxel.

CTV, clinical target volume; HR, high risk; IR, intermediate risk; D90, the minimum dose delivered to $90 \%$ of the target volume; D2cc, minimal dose to $2 \mathrm{~cm}^{3}$.

$C R$, complete remission; $P R$, partial response.

occurred during implantation. There was no grade 3 or 4 late treatment-related toxicity (Table 7).

\section{DISCUSSION}

Since its inception, brachytherapy has played a critical role in the treatment of cervical cancer (9). For patients with parametrial invasion, enhancements in treatment have included IC/IS applicators such as the Vienna applicator (uterine tandem
+ ring) and the Utrecht applicator (uterine tandem + ovoid), with IS needles implanted obliquely to the intrauterine tandem, facilitating good target coverage and resolving the problem of insufficient dose for parametrial diseases $(4,10)$. But the insertion point and planting direction of IS needles were limited by a fixed pinhole position and angle (4). Nomden (10) found that when distal parametrial disease extension was encountered, the HR-CTV could not be completely covered with the IC/IS applicator. Fokdal et al. (11) addressed this distal parametrial disease by the addition of free needles combined with the IC/IS applicator to cover the HR-CTV as much as possible. Because there are few relevant literature reports, the safety and dosimetry of this technology have not been clarified in detail, but it appears to be a clinically feasible treatment option. All 77 brachytherapy fractions enrolled in this study adopted IC/IS+ISBT DP. To investigate whether this technology has a dosimetry advantage over traditional IC/ ISBT, we generated a simulation plan leaving the free needles, and compared it to the original plan. The comparison was limited to single treatment fractions, however, because not all of the brachytherapy fractions of the enrolled patients necessitated the free needles for distal parametrial disease extension. In most patients, free needle insertion was performed to cover the distal parametrial disease extension in the first 1-2 fractions, but owing to tumor shrinkage, conventional IC/ISBT satisfactorily covered the remaining disease. The treatment outcome demonstrated that the addition of free needles to treat distal parametrial disease could significantly improve the coverage area by TV (width, NMD and volume were all significantly increased). Specifically, the contribution to NMD was the most significant, providing an additional treatment width of about $7 \mathrm{~mm}$, which affected the conformity and dose to the HR-CTV. In contrast, if these needles were to be removed, in order to adequately cover the distal parametrial disease area adequately, the thickness of the treatment volume would need to be increased, which would increase the dose delivered to the bladder and rectum, as confirmed by comparison of the relevant dosimetry parameters of the two plans.

There are no reports detailing the optimal application of IC/IS+ ISBT DP. Yoshida et al. (12) explored brachytherapy for different tumor volumes, modeling tumor volumes of 8 groups: $8,12,27$, $36,64,80,96$, and $112 \mathrm{cc}$, managed with four modalities: ICBT, IC/ ISBT and ISBT. The results indicated that the HR-CTV for ISBT and IC/ISBT was better than traditional ICBT when the tumor

TABLE 2 | Comparison of mean values of height, width, thickness, volume and NMD between TV ${ }_{I C / I S+I S B T} D P$ and TV $V_{I C / I S B T}(n=77)$.

\begin{tabular}{|c|c|c|c|}
\hline Parameter & TV IC/IS+ISBT DP & TV IC/ISBT & $P^{*}$ \\
\hline Height (cm) & 7.14 & 7.10 & 0.494 \\
\hline Width (cm) & 6.0 & 5.23 & $<0.001$ \\
\hline Thickness (cm) & 3.71 & 3.82 & 0.027 \\
\hline NMD (cm) & 3.64 & 2.90 & $<0.001$ \\
\hline Volume (cc) & 47.54 & 45.76 & $<0.001$ \\
\hline
\end{tabular}

NMD, the near maximum distance from uterine tandem; TV, treatment volume, target area covered by the $100 \%$ isodose curve.

*Matched pairwise two tailed t-test statistics. 
TABLE 3 | Comparison of dose distribution between IC/IS+ISBT DP and IC/ISBT (Gy, $\alpha \pm S$ ).

\begin{tabular}{|c|c|c|c|c|}
\hline Physical dose & IC/IS+ISBT DP (A) & IC/ISBT (B) & A-B & $\mathbf{P}^{*}$ \\
\hline \multicolumn{5}{|l|}{ HR-CTV } \\
\hline D90 & $8.11 \pm 0.52$ & $7.83 \pm 0.70$ & $0.27 \pm 0.38$ & $<0.001$ \\
\hline D98 & $6.79 \pm 0.56$ & $6.29 \pm 0.84$ & $0.50 \pm 0.53$ & $<0.001$ \\
\hline V100 & $0.966 \pm 0.036$ & $0.949 \pm 0.036$ & $0.017 \pm 0.037$ & $<0.001$ \\
\hline V150 & $0.650 \pm 0.067$ & $0.652 \pm 0.064$ & $-0.002 \pm 0.043$ & 0.686 \\
\hline V200 & $0.370 \pm 0.069$ & $0.394 \pm 0.064$ & $-0.024 \pm 0.042$ & $<0.001$ \\
\hline \multicolumn{5}{|l|}{ IR-CTV } \\
\hline D90 & $4.79 \pm 0.46$ & $4.35 \pm 0.64$ & $0.44 \pm 0.44$ & $<0.001$ \\
\hline Rectem D2cc & $4.15 \pm 0.56$ & $4.38 \pm 0.77$ & $-0.23 \pm 0.44$ & $<0.001$ \\
\hline Bladder D2cc & $5.46 \pm 0.50$ & $5.56 \pm 0.59$ & $-0.1 \pm 0.30$ & 0.007 \\
\hline Sigmoid D2cc & $3.78 \pm 0.83$ & $3.90 \pm 0.94$ & $-0.12 \pm 0.30$ & $<0.001$ \\
\hline small bowel D2cc & $3.55 \pm 0.91$ & $3.57 \pm 0.92$ & $-0.02 \pm 0.18$ & 0.161 \\
\hline
\end{tabular}

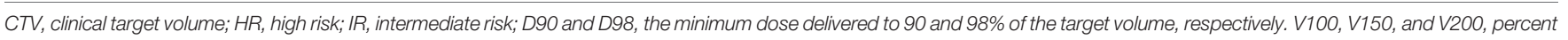
volume of the target receiving at least

100,150 , and $200 \%$ of the prescribed dose, respectively. D2cc, minimal dose to $2 \mathrm{~cm}^{3}$.

*Matched pairwise two tailed t-test statistics.

TABLE 4 | Comparison of HR-CTV D90 with different parametrial extension widths ( $\alpha$ ) between IC/IS+ISBT DP and IC/ISBT (n=77).

\begin{tabular}{|c|c|c|c|c|}
\hline & $\alpha \leq 3 \mathrm{~cm}(n=35)$ & $3 \mathrm{~cm}<\alpha \leq 3.5 \mathrm{~cm}(\mathrm{n}=17)$ & $3.5 \mathrm{~cm}<\alpha \leq 4 \mathrm{~cm}(\mathrm{n}=15)$ & $\alpha>4 \mathrm{~cm}(n=10)$ \\
\hline IC/ISBT D90 (HRCTV) & $8.16 \pm 0.46$ Gy & $7.62 \pm 0.57$ Gy & $7.50 \pm 0.72$ Gy & $7.58 \pm 1.09 \mathrm{~Gy}$ \\
\hline IC/IS+ISBT DP D90 (HRCTV) & $8.20 \pm 0.46$ Gy & $7.96 \pm 0.49$ Gy & $7.98 \pm 0.42$ Gy & $8.25 \pm 0.79$ Gy \\
\hline$P^{*}$ & 0.054 & $<0.001$ & $<0.001$ & 0.002 \\
\hline
\end{tabular}

CTV, clinical target volume; HR, high risk; D90, the minimum dose delivered to $90 \%$ of the target volume.

*Matched pairwise two tailed t-test statistics.

TABLE 5 | Comparison of $D_{2 \mathrm{CC}}$ for OARs with different parametrial extension widths (>3cm and $\left.\leq 3 \mathrm{~cm}\right)$ between IC/IS+ISBT DP and IC/ISBT (Gy, $\alpha \pm S$ ).

\begin{tabular}{|c|c|c|c|}
\hline & IC/IS+ISBT DP (A) & IC/ISBT (B) & $P^{*}$ \\
\hline \multicolumn{4}{|c|}{ Bladder $\mathbf{D}_{2 \mathrm{cc}}$} \\
\hline$\leq 3 \mathrm{~cm}$ & $5.25 \pm 0.51$ & $5.30 \pm 0.52$ & 0.095 \\
\hline$>3 \mathrm{~cm}$ & $5.64 \pm 0.44$ & $5.78 \pm 0.57$ & 0.028 \\
\hline \multicolumn{4}{|c|}{ Rectem $\mathbf{D}_{2 \mathrm{cc}}$} \\
\hline$\leq 3 \mathrm{~cm}$ & $3.89 \pm 0.57$ & $4.02 \pm 0.71$ & 0.027 \\
\hline$>3 \mathrm{~cm}$ & $4.37 \pm 0.45$ & $4.69 \pm 0.70$ & $<0.001$ \\
\hline \multicolumn{4}{|c|}{ Sigmoid $D_{2 c c}$} \\
\hline$\leq 3 \mathrm{~cm}$ & $3.72 \pm 0.74$ & $3.77 \pm 0.75$ & 0.020 \\
\hline$>3 \mathrm{~cm}$ & $3.83 \pm 0.92$ & $4.00 \pm 1.07$ & 0.007 \\
\hline \multicolumn{4}{|c|}{ small bowel $D_{2 c c}$} \\
\hline$\leq 3 \mathrm{~cm}$ & $3.45 \pm 0.99$ & $3.45 \pm 0.98$ & 0.907 \\
\hline$>3 \mathrm{~cm}$ & $3.64 \pm 0.86$ & $3.66 \pm 0.87$ & 0.476 \\
\hline
\end{tabular}

D2cc, minimal dose to $2 \mathrm{~cm}^{3}$.

*Matched pairwise two tailed t-test statistics.

volume was greater than $36 \mathrm{cc}$, and ISBT was significantly better than the other two plans for tumor volumes larger than $80 \mathrm{cc}$. A study by Kirisits et al. (4) showed that the $100 \%$ isodose of ICBT was at maximum $25 \mathrm{~mm}$ from the tandem axis at the level of point $\mathrm{A}$, while the isodose could be moved to $31 \mathrm{~mm}$ when using IC/ISBT. Inspired by these reports, we divided all treatment plans into 4 groups based on parametrial disease extension widths of $\leq 3$, $3.1-3.5,3.6-4$, and $>4 \mathrm{~cm}$ because our results indicated that NMD was significant impacted by parametrial free needles. Compared with IC/ISBT, the dosimetric benefit of IC/IS+ISBT DP was only applicable to parametrial extension widths greater than $3 \mathrm{~cm}$, which is consistent with the conclusion of Kirisits et al. (4). Moreover, the average NMD of all 77 IC/IS+ISBT DP treatment plans in our study could reach $3.64 \mathrm{~cm}$, but only $2.90 \mathrm{~cm}$ after removing the free needles from the distal parametrium. In addition, the V200 of IC/IS+ISBT DP was lower than that of the IC/ISBT group. Although the dwell weight of the free needles was only $16.4 \%$, it still reduced the dwell weight of the needles in the near center area. Therefore, we found it inappropriate to increase free needles in the parametrium just to increase the fitness of 

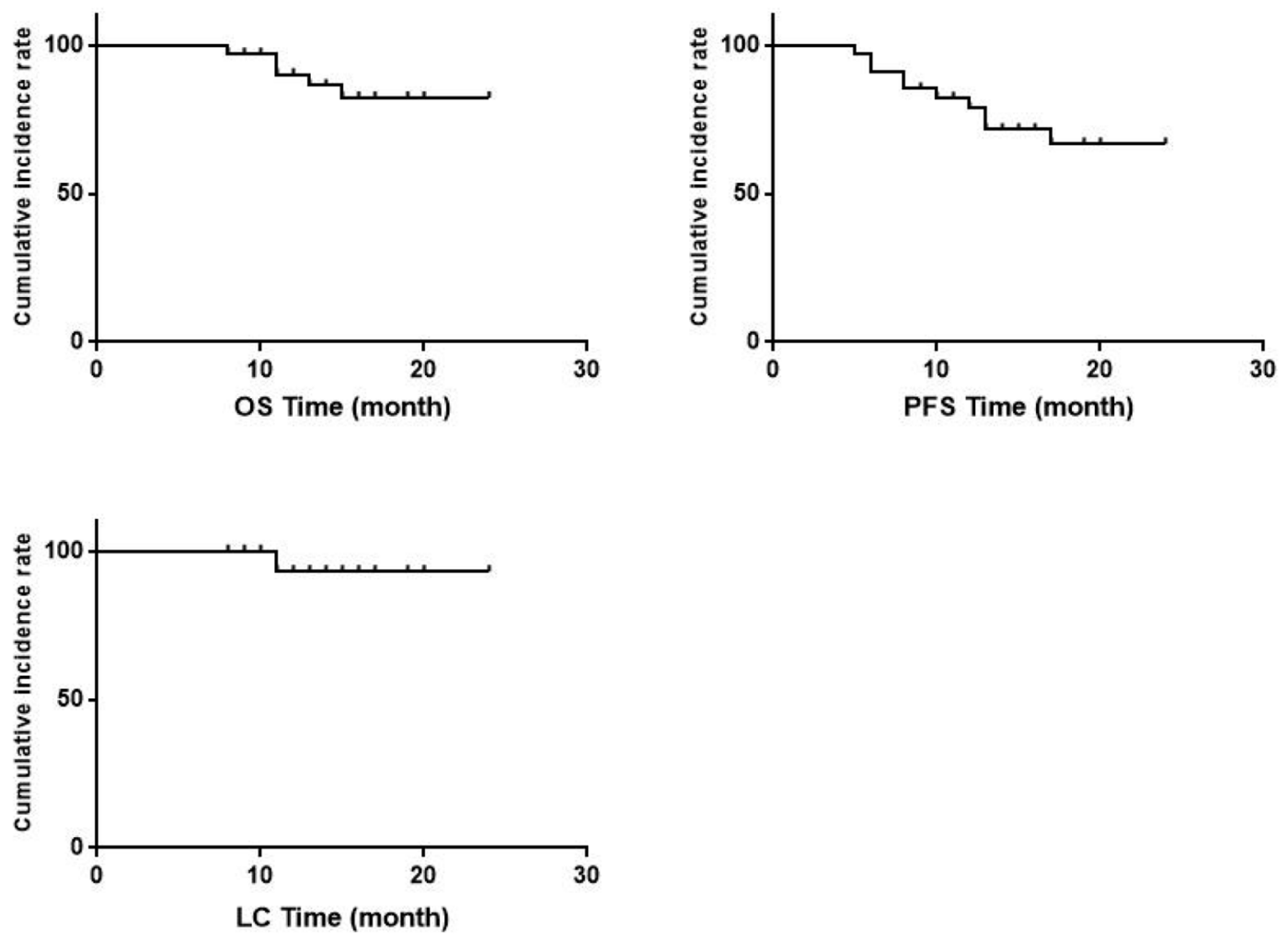

FIGURE 2 | Curves of 2-yr overall survival (OS), 2-yr progression-free survival (PFS) and 2-yr local control rate (LC). No. At Risk parametrial extension widths $<3 \mathrm{~cm}$ 17161616 parametrial extension widths $\geq 3 \mathrm{~cm} 17888$.

TABLE 6 | Prognostic factors on OS and PFS by Cox regression method.

\begin{tabular}{|c|c|c|c|c|}
\hline \multirow[t]{2}{*}{ Variables } & \multicolumn{2}{|c|}{$\begin{array}{c}\text { Overall survival } \\
\text { Univariate analysis }\end{array}$} & \multicolumn{2}{|c|}{$\begin{array}{c}\text { Progression-free survival } \\
\text { Univariate analysis }\end{array}$} \\
\hline & $\mathrm{HR}(95 \% \mathrm{Cl})$ & p & $\mathrm{HR}(95 \% \mathrm{Cl})$ & p \\
\hline age(<50 vs. $\geq 50)$ & $0.41(0.08-2.18)$ & 0.297 & $1.2(0.324-4.441)$ & 0.785 \\
\hline Tumor width( $<5$ vs. $\geq 5 \mathrm{~cm})$ & $1.032(0.20-5.22)$ & 0.969 & $0.285(0.080-1.011)$ & 0.052 \\
\hline Parametrial extension widths $(<3$ vs. $\geq 3 \mathrm{~cm})$ & $0.449(0.089-2.26)$ & 0.331 & $0.14(0.039-0.499)$ & 0.002 \\
\hline Concurrent chemotherapy (no vs. Yes) & $2.337(0.311-17.59)$ & 0.410 & $1.001(0.212-4.713)$ & 0.999 \\
\hline
\end{tabular}

CTV, clinical target volume; $H R$, high risk; D90, the minimum dose delivered to $90 \%$ of the target volume.

$C R$, complete remission, $P R$, partial response,

$H R$, hazard ratio, $\mathrm{Cl}$, confidence interval.

HR-CTV if the IC/ISBT could achieve the dosage requirements, thereby reducing unnecessary trauma by reducing the use of implanted needles. After all, higher V150, and V200 could provide better local control rate (13).

The American Brachytherapy Society recommends 80-90 Gy equivalent doses in 2 Gy fractions (EQD2) for D90 HR-CTV (14). Furthermore, it recommended at least 85 Gy for D90 HRCTV if the tumor measured greater than $4 \mathrm{~cm}$ after EBRT. The
Vienna system study (15) pointed out that when the dose was increased from 81-90 Gy for large tumors, the local control rate increased from $71-90 \%$. A recent study by Dang et al. (16) enrolled 100 cervical cancer patients treated with IC/ISBT. The 5-year LC, DMFS and OS reached 88.9, 81.8, and 77.9\%, respectively. A prospective study (17) enrolled 33 patients with cervical cancer, given ICBT or IC/ISBT, the D90 of HR-CTV reached $88-92.9 \mathrm{~Gy}$, and the 1-year LC and OS were 84 and 


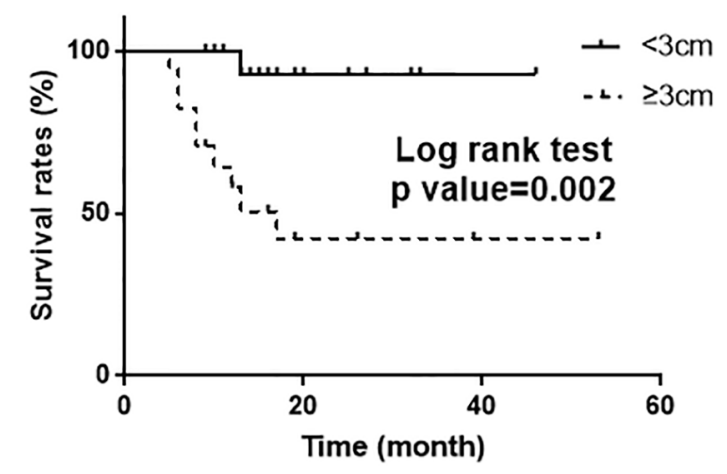

FIGURE 3 | Kaplan-Meier curve of PFS (log rank test $p=0.002$ ) according to the parametrial extension widths.

TABLE 7 | Treatment-related toxicity.

Late toxicity

Value $(n=34)$

Bladder

Grade 0-1

Grade 2

30

Rectum

Grade 0-1

Grade 2

32

2

91.3\%, respectively. But the clinical results of IC/IS+ISBT DP have not been reported. In this study, patients received a median of 91.15GyEQD2 for D90 HR-CTV, which achieved the above dose range. Only 2 cases had local recurrence after 2 years of follow-up, showing an ideal local control rate. Univariate analysis showed that the parametrial extension width is a prognostic risk factor for PFS, indicating that the probability of treatment failure will be significantly increased when the parametrial extension width is greater than $3 \mathrm{~cm}$. Therefore, it is particularly important to give sufficient doses to the distal parametrium. If possible, PET-CT should be used to accurately delineate the target volume for distal parametrium due to the major capability to identify high-risk RT areas (18). For similar patients, even more efforts to devise satisfactory individualized treatment plans will be necessary to address the difficult challenge of simultaneously increasing the dose for HR-CTV yet limiting the dose to OARs.

Previous studies have indicated limiting D2cc $\leq 90 \mathrm{~Gy}$ for the bladder and D2cc $\leq 70$ Gy for the rectum, sigmoid and small bowel (19). The single dose limitation according to our treatment plan indicated a bladder $\mathrm{D}_{2 \mathrm{CC}} \leq 5.88 \mathrm{~Gy}$, and rectum and sigmoid $\mathrm{D}_{2 \mathrm{CC}} \leq 4.98 \mathrm{~Gy}$. In this study, the average dose of OARs were lower than the above-mentioned dose limit, and no grade 3 or 4 bladder LSE or rectum LSE were observed after 2 years follow-up. All the OARs in the 77 IC/IS+ISBT DP plans enrolled in this study were below the above dose limits. The $\mathrm{D}_{2 \mathrm{CC}}$ for rectum and sigmoid for IC/IS+ISBT DP showed little advantage to IC/ISBT when the parametrial extension widths were less than $3 \mathrm{~cm}$. However, the addition of free needles in the distal parametrium significantly contributed to the dose limitation of the bladder, rectum and sigmoid when the parametrial extension widths exceeded $3 \mathrm{~cm}$, especially for the protection of the rectum. In order to cover the area of the distal parametrium as much as possible, the IC/ISBT treatment plan could only increase the dose of the channels closest to the distal parametrium, inevitably leading to an increase in the thickness of the $100 \%$ isodose curve. Therefore, IC/IS+ISBT DP lowered the risk of side effects. In addition, because of the invasive nature of this operation, complications could occur during implantation such as bleeding and infection. The incidence of infections observed in this study was judged acceptable; bleeding due to IS needles was minimal, controlled just with vaginal tamponade. Overall, the patients tolerated IC/IS+ISBT DP well.

At present, ICBT combined with EBRT with parametrial boosting or interstitial brachytherapy (ISBT) have been used by some departments for locally advanced cervical cancer. In order to compare the dose distribution of IC/ISBT and ICBT+ EBRT, Mohamed et al. (20) enrolled 51 patients with locally advanced cervical cancer from 2008 to 2011 . The results showed that the HR-CTV D90 provided by the IC/ISBT group was significantly higher than that of the ICBT+EBRT group (the HR-CTV D90 of 3 cases in the ICBT+EBRT group was less than $79 \mathrm{~Gy}$, while all patients in the IC/ISBT group were higher than 84 Gy). Additionally, the IC/ISBT group also had obvious advantages regarding $\mathrm{D}_{2 \mathrm{CC}}$ of OARs. Furthermore, EBRT could not be synthesized with brachytherapy perfectly, leading to omission and overlap of doses, and inaccurate dose assessment. Lindegaard et al. (21) also pointed out that parametrial boosting by EBRT could only provide doses to the HR-CTV and IR-CTV selectively, with consequent significantly increased radiation dose to OARs. The conclusion: for cervical cancer with associated parametrial disease extension, EBRT supplementation cannot replace brachytherapy. ISBT, either by freehand or template implantation, is a preferred treatment option for locally advanced cervical cancer. The angle and position of the implant needles were unlimited, and it could provide excellent DVH parameters (22). However, Hsu et al. (23) pointed out that although ISBT has a high degree of conformity to the HR-CTV, the treatment volume receiving $>180 \%$ of the prescribed dose was significantly lower than ICBT (17 vs 31cc). Although there are no direct comparison reports, previous studies have shown that the size of the central high-dose area could affect the local control rate (13). In addition, the precisely accurate location and reproducibility of ISBT with a free hand technique was limited. IC/IS+ISBT DP minimizes the use of free needles and improves accuracy and reproducibility. Takahiro et al. (24) compared dosimetric differences between IC/ISBT and ISBT, and the results showed that the V100 of IC/ISBT to be minimally different from ISBT. Therefore, we contend that the results of this and other studies demonstrate that IC/IS+ISBT DP has advantages in dosimetry and treatment accuracy for cervical cancer complicated by distal parametrial disease extension compared with ISBT. 
In summary, the evidence indicates that the radiotherapy technique of IC/IS+ISBT DP has excellent dosimetry parameters for cervical cancer patients with distal parametrial extension (particularly parametrial extension widths greater than $3 \mathrm{~cm}$ ). However, although brachytherapy appears effective and valuable, especially in gynecological malignancies, and cannot be replaced by intensity modulated or stereotactic radiotherapy, it has been progressive decline over the past decades. And the development of brachytherapy technology is relatively backward compared with external beam radiotherapy. It is necessary to strengthen the clinical practice, education, research and communication (25). Practical education should be given special attention due to the higher technical requirements for the operator, especially the technology mentioned in our research. It is recommended to set up more brachytherapy training institutions, aiming to educate physicians/medical physicists in brachytherapy through a multidisciplinary approach such as GEMELLI-INTERACTS (Interventional Radiotherapy Active Teaching School) mode (26), to better serve patients.

\section{DATA AVAILABILITY STATEMENT}

The raw data supporting the conclusions of this article will be made available by the authors, without undue reservation.

\section{REFERENCES}

1. Rose PG, Bundy BN, Watkins EB, Thigpen JT, Deppe G, Maiman MA, et al. Concurrent cisplatin-based radiotherapy and chemotherapy for locally advanced cervical cancer. N Engl J Med (1999) 340(15):1144-53. doi: 10.1056/NEJM199904153401502

2. Tanderup K, Eifel PJ, Yashar CM, Pötter R, Grigsby PW. Curative radiation therapy for locally advanced cervical cancer: brachytherapy is NOT optional. Int J Radi Oncol Biol Phys (2014) 88(3):537-9. doi: 10.1016/j.ijrobp. 2013.11.011

3. Zhao Z, Zhang N, Liu Y, Wu N, Mao Z, Yang W, et al. Analysis of clinical utilization of ring applicator for combined intracavitary/interstitial imageguided brachytherapy treatment in Chinese patients with locally advanced cervical cancer. J Contemp Brachyther (2020) 12(3):252. doi: 10.5114/ jcb.2020.96866

4. Kirisits C, Lang S, Dimopoulos J, Berger D, Georg D, Pötter R. The Vienna applicator for combined intracavitary and interstitial brachytherapy of cervical cancer: design, application, treatment planning, and dosimetric results. Int J Radi Oncol Biol Phys (2006) 65(2):624-30. doi: 10.1016/ j.ijrobp.2006.01.036

5. Haie-Meder C, Pötter R, Van Limbergen E, Briot E, De Brabandere M, Dimopoulos J, et al. Recommendations from Gynaecological (GYN) GECESTRO Working Group(I): concepts and terms in 3D image based 3D treatment planning in cervix cancer brachytherapy with emphasis on MRI assessment of GTV and CTV. Radiother Oncol (2005) 74(3):235-45. doi: 10.1016/j.radonc.2004.12.015

6. Viswanathan AN, Erickson B, Gaffney DK, Beriwal S, Bhatia SK, Burnett Iii $\mathrm{OL}$, et al. Comparison and consensus guidelines for delineation of clinical target volume for CT-and MR-based brachytherapy in locally advanced cervical cancer. Int J Radi Oncol Biol Phys (2014) 90(2):320-8. doi: 10.1016/ j.ijrobp.2014.06.005

7. Kirisits C, Pötter R, Lang S, Dimopoulos J, Wachter-Gerstner N, Georg D. Dose and volume parameters for MRI-based treatment planning in intracavitary brachytherapy for cervical cancer. Int J Radiat Oncol Biol Phys (2005) 62(3):901-11. doi: 10.1016/j.ijrobp.2005.02.040

\section{ETHICS STATEMENT}

Written informed consent was obtained from the individual(s) for the publication of any potentially identifiable images or data included in this article.

\section{AUTHOR CONTRIBUTIONS}

H-DQ performed the statistical analysis and drafted the manuscript. G-HC participated in the design of this study and performed manuscript review. D-MH and NZ performed patients follow-up and data collection. ZM devised the treatment plan. All authors contributed to the article and approved the submitted version.

\section{FUNDING}

This work was partially supported by grants from the National Natural Science Foundation of China [grant numbers 82073331, 81201737, 31600679,81703034]; Project of Science and Technology Department of Jilin Province (grant number 20190303151SF);Horizontal Project of Jilin University [grant numbers 2019YX435, 2019155].

8. Cox JD, Stetz J, Pajak TF. Toxicity criteria of the radiation therapy oncology group (RTOG) and the European organization for research and treatment of cancer (EORTC). Int J Radi Oncol Biol Phys (1995) 31(5):1341-6. doi: 10.1016/0360-3016(95)00060-C

9. Mould RF. "The historical roots of modern brachytherapy for cervical and endometrial cancer." In: Vahrson H.W. (Ed.) Radiat Oncol Gynecol Cancers. Berlin, Heidelberg. FL: Springer (1997) 1-9 doi: 10.1007/978-3-64260334-1_1

10. Nomden CN, de Leeuw AAC, Moerland MA, Roesink JM, Tersteeg RJHA, Jürgenliemk-Schulz IM. Clinical use of the Utrecht applicator for combined intracavitary/interstitial brachytherapy treatment in locally advanced cervical cancer. Int J Radi Oncol Biol Phys (2012) 82(4):1424-30. doi: 10.1016/ j.ijrobp.2011.04.044

11. Fokdal L, Tanderup K, Hokland SB, Røhl L, Pedersen EM, Nielsen SK, et al. Clinical feasibility of combined intracavitary/interstitial brachytherapy in locally advanced cervical cancer employing MRI with a tandem/ring applicator in situ and virtual preplanning of the interstitial component. Radiother Oncol (2013) 107(1):63-8. doi: 10.1016/j.radonc.2013.01.010

12. Yoshida K, Yamazaki H, Kotsuma T, Takenaka T, Ueda MM, Miyake S, et al. Simulation analysis of optimized brachytherapy for uterine cervical cancer: Can we select the best brachytherapy modality depending on tumor size? Brachytherapy (2016) 15(1):57-64. doi: 10.1016/j.brachy.2015.10.002

13. Murakami N, Kobayashi K, Shima S, Tsuchida K, Kashihara T, Tselis N, et al. A hybrid technique of intracavitary and interstitial brachytherapy for locally advanced cervical cancer: initial outcomes of a single-institute experience. BMC Cancer (2019) 19(1):221. doi: 10.1186/s12885-019-5430-x

14. Viswanathan AN, Thomadsen B. American Brachytherapy Society Cervical Cancer Recommendations C. American Brachytherapy Society consensus guidelines for locally advanced carcinoma of the cervix. Part I: general principles. Brachytherapy (2012) 11(1):33-46. doi: 10.1016/j.brachy. 2011.07.003

15. Pötter R, Dimopoulos J, Georg P, Lang S, Waldhäusl C, Wachter-Gerstner N, et al. Clinical impact of MRI assisted dose volume adaptation and dose escalation in brachytherapy of locally advanced cervix cancer. Radiother Oncol (2007) 83(2):148-55. doi: 10.1016/j.radonc.2007.04.012 
16. Dang Y-Z, Li P, Li J-P, Bai F, Zhang Y, Mu Y-F, et al. The efficacy and late toxicities of computed tomography-based brachytherapy with intracavitary and interstitial technique in advanced cervical cancer. J Cancer (2018) 9 (9):1635. doi: 10.7150/jca.23974

17. Harkenrider MM, Surucu M, Harmon G, Mysz ML, Shea SM, Yacoub J, et al. Early outcomes and impact of a hybrid IC/IS applicator for a new MRI-based cervical brachytherapy program. Brachytherapy (2018) 17(1):187-93. doi: 10.1016/j.brachy.2017.09.010

18. Fiorentino A, Laudicella R, Ciurlia E, Annunziata S, Lancellotta V, Mapelli P, et al. Positron emission tomography with computed tomography imaging (PET/CT) for the radiotherapy planning definition of the biological target volume: PART 2. Crit Rev Oncol Hematol (2019) 139:117-24. doi: 10.1016/ j.critrevonc.2019.03.008

19. Levitchi M, Charra-Brunaud C, Quetin P, Haie-Meder C, Kerr C, Castelain B, et al. Impact of dosimetric and clinical parameters on clinical side effects in cervix cancer patients treated with $3 \mathrm{D}$ pulse-dose-rate intracavitary brachytherapy. Radiother Oncol (2012) 103(3):314-21. doi: 10.1016/ j.radonc.2012.04.008

20. Mohamed S, Kallehauge J, Fokdal L, Lindegaard JC, Tanderup K. Parametrial boosting in locally advanced cervical cancer: combined intracavitary/ interstitial brachytherapy vs. intracavitary brachytherapy plus external beam radiotherapy. Brachytherapy (2015) 14(1):23-8. doi: 10.1016/ j.brachy.2014.09.010

21. Lindegaard JC, Tanderup K. Counterpoint: time to retire the parametrial boost. Brachytherapy (2012) 11(2):80-3. doi: 10.1016/j.brachy.2012. 01.004

22. Saitoh J-i, Ohno T, Sakurai H, Katoh H, Wakatsuki M, Noda S-e, et al. Highdose-rate interstitial brachytherapy with computed tomography-based treatment planning for patients with locally advanced uterine cervical carcinoma. J Radiat Res (2011) 52(4):490-5. doi: 10.1269/jrr.10189
23. Hsu ICJ, Speight J, Hai J, Vigneault E, Phillips T, Pouliot J. A comparison between tandem and ovoids and interstitial gynecologic template brachytherapy dosimetry using a hypothetical computer model. Int J Radi Oncol Biol Phys (2002) 52(2):538-43. doi: 10.1016/S0360-3016(01)02691-8

24. Oike T, Ohno T, Noda S-e, Kiyohara H, Ando K, Shibuya K, et al. Can combined intracavitary/interstitial approach be an alternative to interstitial brachytherapy with the Martinez Universal Perineal Interstitial Template (MUPIT) in computed tomography-guided adaptive brachytherapy for bulky and/or irregularly shaped gynecological tumors? Radiother Oncol (2014) 9 (1):222. doi: 10.1186/s13014-014-0222-6

25. Tagliaferri L, Vavassori A, Lancellotta V, De Sanctis V, Barbera F, Fusco V, et al. Can brachytherapy be properly considered in the clinical practice? Trilogy project: The vision of the AIRO (Italian Association of Radiotherapy and Clinical Oncology) Interventional Radiotherapy study group. J Contemp Brachyther (2020) 12(1):84. doi: 10.5114/jcb.2020.92765

26. Kovács G, Tagliaferri L, Valentini V. Is an Interventional Oncology Center an advantage in the service of cancer patients or in the education? The Gemelli Hospital and INTERACTS experience. J Contemp Brachyther (2017) 9(6):497. doi: $10.5114 /$ jcb.2017.72603

Conflict of Interest: The authors declare that the research was conducted in the absence of any commercial or financial relationships that could be construed as a potential conflict of interest.

Copyright (c) $2021 \mathrm{Qu}, \mathrm{Han}$, Zhang, Mao and Cheng. This is an open-access article distributed under the terms of the Creative Commons Attribution License (CC BY). The use, distribution or reproduction in other forums is permitted, provided the original author(s) and the copyright owner(s) are credited and that the original publication in this journal is cited, in accordance with accepted academic practice. No use, distribution or reproduction is permitted which does not comply with these terms. 